\title{
Erratum to: Normal subgroups of the algebraic fundamental group of affine curves in positive characteristic
}

\author{
Amílcar Pacheco • Katherine F. Stevenson • \\ Pavel Zalesskii
}

Published online: 17 May 2014

(C) Springer-Verlag Berlin Heidelberg 2014

\section{Erratum to: Math. Ann. (2009) 343:463-486 DOI 10.1007/s00208-008-0279-3}

Let $k$ be an algebraically closed field of characteristic $p>0$ of countable cardinality. Denote by $C$ a smooth, connected, affine curve defined over $k$ with function field $K$ and by $\pi_{1}(C)$ its algebraic fundamental group. In the original paper we addressed the question of the structure of the normal closed subgroups of $\pi_{1}(C)$. More precisely, in Theorem 1.1, we gave a sufficient condition for such a normal closed subgroup $N$ to be isomorphic to a subgroup of a free profinite group of countable rank. There we claimed to prove this under the hypothesis that the group $M=\pi_{1}(C) / N$ had an infinitely generated Sylow $p$-subgroup.

However, as remarked in the paragraph after Corollary 1.2 of [1], this hypothesis is not enough. We would like to make this remark more explicit by stating (as noticed by those authors) the additional hypothesis needed to make Theorem 1.1 work. For each integer $g \geq 0$ denote by $P_{g}(C)$ the intersection of algebraic fundamental groups

The online version of the original article can be found under doi:10.1007/s00208-008-0279-3.

\section{A. Pacheco $(\square)$}

Instituto de Matemática, Universidade Federal do Rio de Janeiro,

Rua Alzira Brandão 355/404, Tijuca, Rio de Janeiro, RJ 20550-035, Brazil

e-mail: amilcar@acd.ufrj.br

\section{K. F. Stevenson}

Department of Mathematics, California State University, Northridge, CA 91330, USA

e-mail: katherine.stevenson@csun.edu

\section{P. Zalesskii}

Departamento de Matemática, Universidade de Brasília, Brasília, DF 70910-900, Brazil

e-mail: pz@mat.unb.br 
of étale $\mathbb{Z} / p$-covers $Z \rightarrow C$ such that the genus of the smooth compactification of $Z$ is at least $g$. This is a subgroup of $\pi_{1}(C)$. Recall that if $\bar{C}$ denotes the smooth compactification of $C$ and $S=\bar{C} \backslash C$, then $\pi_{1}(C)$ is isomorphic to $\operatorname{Gal}\left(K_{\mathrm{un}, S} / K\right)$, where $K_{\text {un }, S}$ denotes the maximal Galois extension of $K$ which is étale over $C$. In this context the subfield $K_{g}(C)$ of $K_{\text {un }, S}$ corresponding to $P_{g}(C)$ determines an pro-cover $Z_{g}(C) \rightarrow C$ which dominates every étale $\mathbb{Z} / p$-cover of $C$.

Hypothesis 1 Suppose that the normal subgroup $N$ is contained in $P_{g}(C)$ for some integer $g \geq 0$.

Denote by $K_{M}$ the subfield of $K_{\text {un }, S}$ fixed by $N$. Under the previous hypothesis we have $K_{g}(C) \subset K_{M}$. We change the construction of Lemma 4.5 in the original paper by that of [1, Lemma 2.2]. This gives a $\mathbb{Z} / p$-cover $T \times_{k[[t]]} k((t))$ of $\bar{C} \times_{k} k((t))$ which is étale over $C$. In particular, specializing it to the closed fiber, this will give a $\mathbb{Z} / p$-cover $T_{s}$ of $\bar{C}$ which is étale over $C$. The algebraic fundamental group of this curve and its map to $G^{\prime}$ and $\Gamma^{\prime}$ will give a solution to the embedding problem (4.4) in the original paper. Furthermore, in contrast with the solution obtained in the original article, the function field of $T_{S}$ will be contained in $K_{M}$ (since $K_{M}$ contains $K_{g}(C)$ for some integer $g \geq 0$ ). This fixes the gap in the original article.

Observe finally that we could have proved Theorem 1.1 in the original paper without the hypothesis on the Sylow $p$-subgroup, but with the previous hypothesis. This is covered by [1, Corollary1.2].

Acknowledgments We would like to thank the authors of [1] for their private communication on the subject.

\section{Reference}

1. Bary-Soroker, L., Kumar, M.: Subgroup structure of fundamental groups in positive characteristic. Commun. Algebra 41, 3705-3719 (2013) 